\title{
An MDM2 inhibitor achieves synergistic cytotoxic effects with adenoviruses lacking E1B55kDa gene on mesothelioma with the wild-type $p 53$ through augmenting NFI expression
}

Thao Thi Thanh Nguyen ${ }^{1,2,3}$, Masato Shingyoji ${ }^{4}$, Michiko Hanazono ${ }^{1,5}$, Boya Zhong ${ }^{1,2}$, Takao Morinaga ${ }^{1}$, Yuji Tada $^{6,7}$, Hideaki Shimada $^{8}$, Kenzo Hiroshima ${ }^{5,9}$ and Masatoshi Tagawa (iD) ${ }^{1,5,10 凶}$

(C) The Author(s) 2021

A majority of mesothelioma specimens were defective of $\mathrm{p} 14$ and $\mathrm{p} 16$ expression due to deletion of the INK4A/ARF region, and the p53 pathway was consequently inactivated by elevated MDM2 functions which facilitated p53 degradaton. We investigated a role of p53 elevation by MDM2 inhibitors, nutlin-3a and RG7112, in cytotoxicity of replication-competent adenoviruses (Ad) lacking the $\mathrm{p} 53$-binding E1B55kDa gene (Ad-delE1B). We found that a growth inhibition by p53-activating Ad-delE1B was irrelevant to p53 expression in the infected cells, but combination of Ad-delE1B and the MDM2 inhibitor produced synergistic inhibitory effects on mesothelioma with the wild-type but not mutated $p 53$ genotype. The combination augmented p53 phosphorylation, activated apoptotic but not autophagic pathway, and enhanced DNA damage signals through ATM-Chk2 phosphorylation. The MDM2 inhibitors facilitated production of the Ad progenies through augmented expression of nuclear factor I (NFI), one of the transcriptional factors involved in Ad replications. Knocking down of p53 with siRNA did not increase the progeny production or the $\mathrm{NFI}$ expression. We also demonstrated anti-tumor effects by the combination of Ad-delE1B and the MDM2 inhibitors in an orthotopic animal model. These data collectively indicated that upregulation of wild-type p53 expression contributed to cytotoxicity by E1B55kDa-defective replicative Ad through NFI induction and suggested that replication-competent Ad together with augmented p53 levels was a therapeutic strategy for $p 53$ wild-type mesothelioma.

Cell Death and Disease (2021)12:663; https://doi.org/10.1038/s41419-021-03934-y

\section{INTRODUCTION}

Mutations of $p 53$ gene were infrequent in mesothelioma but a majority of the clinical specimens did not express p14 or p16 due to either deletion of the encoding CDKN2A locus or methylation of the transcriptional regulatory regions [1]. Recent genome-wide studies also confirmed the CDKN2A deletion and loss of p53 functions in the majority of mesothelioma despite having the wild-type $p 53$ genotype $[2,3]$. The loss of $p 14$ expression resulted in enhanced MDM2 activity, which mediated ubiquitination and subsequent degradation of $\mathrm{p} 53$. The lack of $\mathrm{p} 16$ led to activation of cyclin-dependent kinase $4 / 6$, which increased pRb phosphorylation and promoted cell cycle progression. Most of the mesothelioma are therefore defective of these tumor suppressor functions. The characteristic genetic alterations suggested that p53 re-expression played a crucial role in a therapeutic strategy for mesothelioma [4]. In fact forced expression of p53 in mesothelioma not only activated p53-mediated cell death pathways but dephosphorylated pRb since p21, one of the p53 targets, blocked cyclin-dependent kinase 2 [5]. These data collectively indicated that the p53 downstream pathways were intact in mesothelioma and that restoration of $\mathrm{p} 53$ expression was the therapeutic options.

Replication-competent adenoviruses (Ad) induced cytotoxicity preferentially in tumors and were tested for the clinical feasibility [6]. Nevertheless, a role of p53 in the Ad-mediated cytotoxicity was not well understood. Upregulated p53 levels facilitated death of Ad-infected cells and then decreased production of the cytotoxic virus progenies $[7,8]$, whereas elevated p53 promoted tumor cell death [9]. Ad replication by itself influenced endogenous p53 expression in the infected cells. E1A molecules increased a p53 level, but E1B55kDa inactivated p53 [10]. We therefore examined effects of Ad defective of the gene encoding E1B55kDa molecules (Ad-delE1B) on mesothelioma with the wild-type $p 53$ genotype, and demonstrated that Ad-delE1B upregulated p53 expression

\footnotetext{
${ }^{1}$ Division of Pathology and Cell Therapy, Chiba Cancer Center Research Institute, 666-2 Nitona, Chuo-ku, Chiba 260-8717, Japan. ${ }^{2}$ Department of Molecular Biology and Oncology, Graduate School of Medicine, Chiba University, 1-8-1 Inohana, Chuo-ku, Chiba 260-8670, Japan. ${ }^{3}$ Division of Medical Biotechnology, Biotechnology Center of Ho Chi Minh City, 2374 National Highway 1, District 12, Ho Chi Minh, Vietnam. ${ }^{4}$ Division of Respirology, Chiba Cancer Center, 666-2 Nitona, Chuo-ku, Chiba 260-8717, Japan. ${ }^{5}$ Department of Biochemistry and Genetics, Graduate School of Medicine, Chiba University, 1-8-1 Inohana, Chuo-ku, Chiba 260-8670, Japan. ${ }^{6}$ Department of Respirology, Graduate School of Medicine, Chiba University, 1-8-1 Inohana, Chuo-ku@, Chiba 260-8670, Japan. ${ }^{7}$ Department of Respiratory Medicine, International University of Health and Welfare Atami Hospital, 13-1 Higasikaigan, Atami 413-0012, Japan. ${ }^{8}$ Department of Surgery, Graduate School of Medicine, Toho University, 6-11-1 Oomori-nishi, Oota-ku 143-8541 Tokyo, Japan.

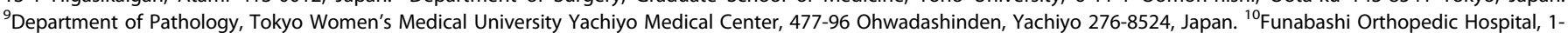

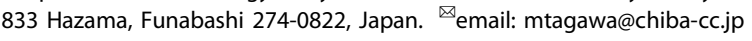
Edited by: G. Blandino 
and produced synergistic cytotoxicity with cisplatin [11]. These data suggested that increased p53 expression was favorable for cytotoxicity induced by Ad and a DNA damaging agent.

Expression of p53 is regulated not only at the transcriptional level but in the ubiquitination process. Ubiquitinated p53 is subjected to proteasome-mediated degradation and MDM2 plays a major role in the process. An agent to inhibit the MDM2 function can consequently increase endogenous p53 levels. An imidazole compound such as nutlin-3a and RG7112 functions as an MDM2 inhibitor, upregulated p53 levels and activated p53-mediated apoptosis in tumors with the wild-type $p 53$ genotype $[12,13]$.

In this study, we examined how p53 upregulation by Ad-delE1B contributed to the Ad-mediated cytotoxicity, and investigated effects of MDM2 inhibitors on Ad-delE1B-mediated cell death and the viral replications. The present study demonstrated a role of p53 in activation of DNA damage signals and a cellular factor involved in Ad replications.

\section{MATERIALS AND METHODS \\ Cells, agents, and mice}

Mesothelioma with the wild-type $p 53, \mathrm{MSTO}-211 \mathrm{H}, \mathrm{NCl}-\mathrm{H} 226$, and $\mathrm{NCl}-\mathrm{H} 28$ cells, were obtained from ATCC, and those with mutated $p 53$, EHMES-1 and JMN-1B cells, were from Dr. Hamada (Hiroshima University, Japan), and A549 lung carcinoma cells were from Cell Resource Center for Biomedical Research (Sendai, Japan). All the p53 wild-type mesothelioma cells were defective of the CDKN2A locus. Nutlin-3a was obtained from Selleck (Houston, TX), and RG7112 from Roche TCRC (New York, NY) or MedChem (Monmouth Junction, NJ). BALB/C nu/nu mice were purchased from SLC (Hamamatsu, Japan). Cells were negative for mycoplasma and authorized by STR analysis.

\section{Ad preparations}

Replication-competent Ad-delE1B, Ad-p53 or Ad expressing $\beta$-galactosidase gene (Ad-LacZ) were prepared with an Adeno- $X$ expression system (Takara, Shiga, Japan). Virus particles (vp) were estimated with the formula: absorbance at $260 \mathrm{~nm}$ in $0.1 \%$ sodium dodecyl sulfate $\times 1.1 \times 10^{12}$.

\section{Western blot analysis}

Cell lysate was subjected to electrophoresis, transferred onto a nylon filter and hybridized with antibody (Ab) against phosphorylated p53 at Ser 15, p21, cleaved capase-3, poly-(ADP ribose) polymerase (PARP) (that also detects cleaved PARP), cleaved caspase-8 at Asp381, Fas, FADD, cleaved caspase-9, Bax, PUMA, Atg-5, Beclin-1, LC3A/B, Chk2, ATR, phosphorylated Chk1 at Ser 345, phosphorylated Chk2 at Thr68, H2AX, actin (Cell Signaling, Danvers, MA), MDM2, E1A, Chk1, B23, TFIID, NFI (Santa Cruz, Dallas, TX), Oct1, ATM (Millipore, Temecula, CA), phosphorylated ATM at Ser 1981, KAP1, phosphorylated KAP1 at Ser 824 (Abcam, Cambridge, UK), phosphorylated H2AX at Ser $139(\gamma-\mathrm{H} 2 \mathrm{AX})$ (BioLegend, San Diego, CA), p53, phosphorylated ATR at Ser 428 (Thermo Fisher, Fremont, $(A)$.

\section{RNA interference and virus production}

Cells were transfected with siRNA targeting p53 (\#TP53-VHS40367) or nonspecific siRNA (\#12935-114) (Thermo Fisher) as a control using Lipofectamine RNAiMAX according to the manufacturer's protocol (Thermo Fisher). For detecting viral production, cells were treated with Ad-delE1B and/or MDM2 inhibitors, and the lysate was examined for cytotoxicity with A549 cells. The virus titers were calculated with the $\mathrm{TCID}_{50}$ method.

\section{Cell viability assay}

Cells were treated with Ad-delE1B for 4 days or MDM2 inhibitors for 2 days. For combination treatments, cells were infected with Ad-delE1B for 2 days and then treated with MDM2 inhibitors for further 2 days. Cell viability was assayed with a cell-counting WST kit (Wako, Osaka, Japan), (WST assay). Combinatory effects were examined by CalcuSyn software (Biosoft, Cambridge, UK). Combination index (Cl) values at respective fraction affected ( $\mathrm{Fa}$ ) points which showed relative levels of suppressed cell viability were calculated based on the WST assay. $\mathrm{Cl}<1, \mathrm{Cl}=1$ and $\mathrm{Cl}>1$ indicate synergistic, additive, and antagonistic actions, respectively. Viable cell numbers were counted with the trypan blue dye exclusion test (Wako).

\section{Cell cycle analysis and apoptosis}

Cells treated with RNase and propidium iodide (PI) $(50 \mu \mathrm{g} / \mathrm{ml})$ were analyzed for the cell cycle with FACSCalibur (BD, San Diego, CA). Cells were also stained with annexin V Alexa Fluor 488 and PI, and analyzed for apoptosis with an image-based cytometer (Invitrogen, Carlsbad, CA).

\section{Immunofluorescence}

Cells treated with $4 \%$ paraformaldehyde, $0.25 \%$ Triton X-100 and 5\% bovine serum albumin were incubated with anti-p53 or anti-NFI Ab and secondary Ab conjugated with Alexa Fluor 488 or Alexa Fluor 555. Nuclei were stained with 4',6-diamidine-2-phenylindole (DAPI) $(1 \mu \mathrm{g} / \mathrm{ml})$ or $\mathrm{PI}$ $(50 \mu \mathrm{g} / \mathrm{ml})$. The images were analyzed with a laser confocal microscope (Leica, Deerfield, IL).

\section{Animal studies}

All animal experiments have been performed according to the guideline of Chiba University and were permitted from the animal experiment committee (permission number: A2-160 and A2-161). Four-week-old BALB/c nu/nu mice were purchased from SLC (Hamamatsu, Japan). All mice were housed in clean conditions with soft food and water in the animal resource center at Chiba University. These mice were randomly divided into eight groups (6 mice/group). BALB/c nude mice were anesthetized with pentobarbital sodium $(1 \%, 0.01 \mathrm{ml} / \mathrm{g})$ (Sigma-Aldrich, Darmstadt, Germany) before injected with MSTO- $211 \mathrm{H}$ cells $\left(5 \times 10^{6}\right.$ cells $)$ into the pleural cavity on day 1, treated with an intrapleural injection of $\mathrm{Ad}$ on day 4 , and with an intraperitoneal injection of MDM2 inhibitors on day 10. Mice were measured body weight every 2 days. Mice were sacrificed by cervical dislocation after being anesthetized on day 25 . Xenografts were harvested and tumor weights were measured.

\section{Statistical analyses}

All data are presented as the mean $\pm \mathrm{SE}$. One-way analysis of variance followed by post hoc Tukey's test was used to assess significant differences between two groups or more. $p$ value $<0.05$ was considered statistically significant. Statistical analyses were performed using GraphPad Prism 6 software (GraphPad software, La Jolla, CA, USA).

\section{RESULTS}

Ad-delE1B induced p53-independent growth suppression We investigated growth inhibitory effects of Ad-delE1B on mesothelioma with wild-type (MSTO-211H, NCl-H226 and $\mathrm{NCl}-$ $\mathrm{H} 28$ ) or mutated $p 53$ genotype (EHMES-1 and JMN-1B) (Fig. 1A). All cells were susceptible to Ad-delE1B but not to Ad-LacZ as a control. These cells showed similar $I C_{50}$ for inhibition, suggesting that Ad-delE1B-mediated effects were irrelevant to the $p 53$ genotype. We also examined p53 expression and the phosphorylation by Ad-delE1B (Fig. 1B). The expression and phosphorylation at Ser 15 were upregulated in the wild-type $p 53$ cells, whereas those in mutated cells remained unchanged or decreased. These data indicated that Ad-delE1B increased endogenous wild-type p53 levels but the upregulated expression did not contribute to growth inhibition.

We examined a role of p53 in the Ad-delE1B-induced growth suppression by knocking down p53 (Fig. 1C). Cells treated with p53 siRNA eliminated p53 expression irrespective of p53 genotypes and the $p 53$ levels remained suppressed even after Ad-delE1B infection. Susceptibility of p53 siRNA-treated cells to Ad-delE1B was not different from that of cells untreated or treated with control siRNA regardless of the $p 53$ genotype. These data indicated that Ad-delE1B-induced cytotoxicity was independent of p53 levels and increased p53 expression by Ad-delE1B did not influence the cytotoxicity.

\section{MDM2 inhibitors-mediated growth suppression}

We examined susceptibility of mesothelioma to MDM2 inhibitors, nutlin-3a and RG7112 (Fig. 2A, Supplementary Table 1). Both inhibitors achieved inhibitory effects to mesothelioma with wildtype greater than those with mutated $p 53$ genotype. Average $\mathrm{IC}_{50}$ values of nutlin-3a to wild-type $p 53$ cells $(4.07 \pm 0.59 \mu \mathrm{M})$ was 


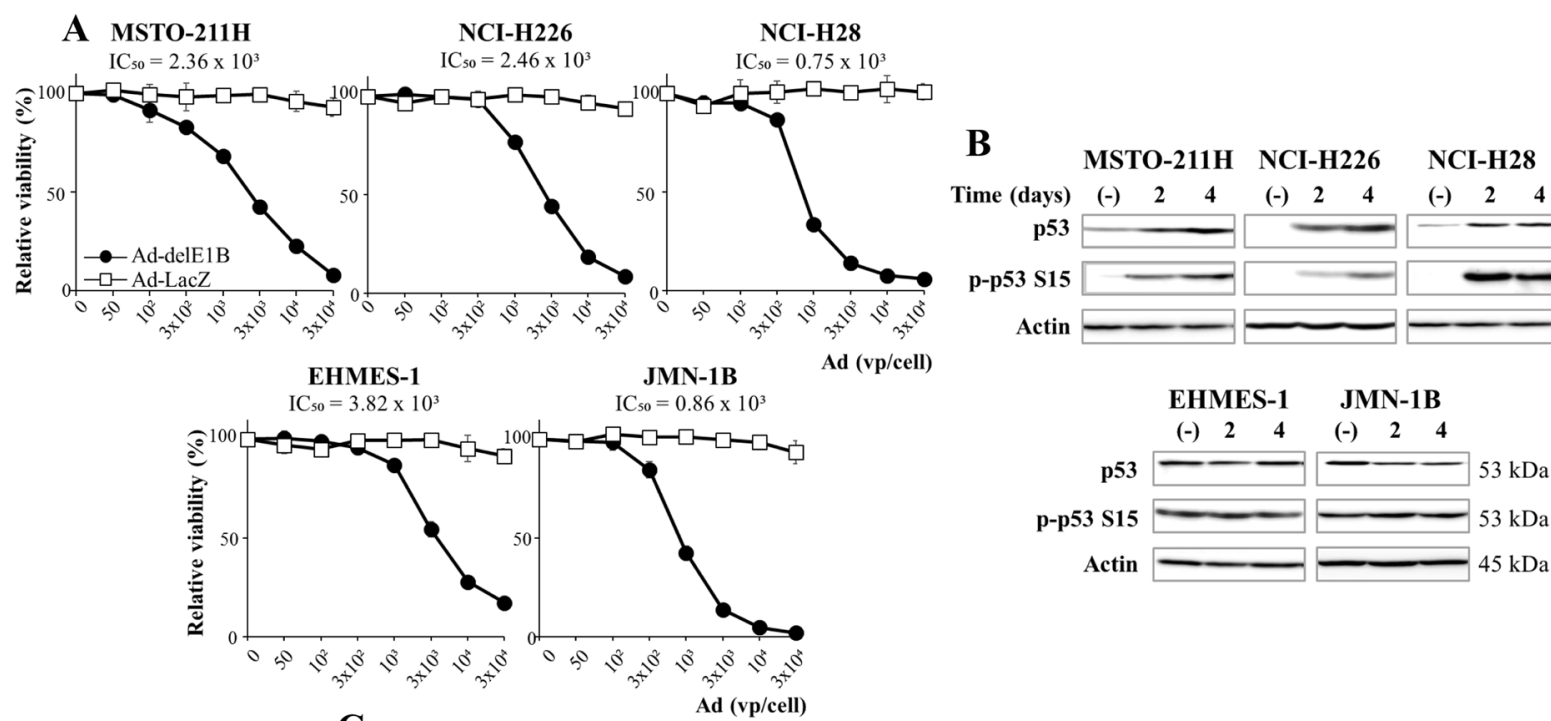

C

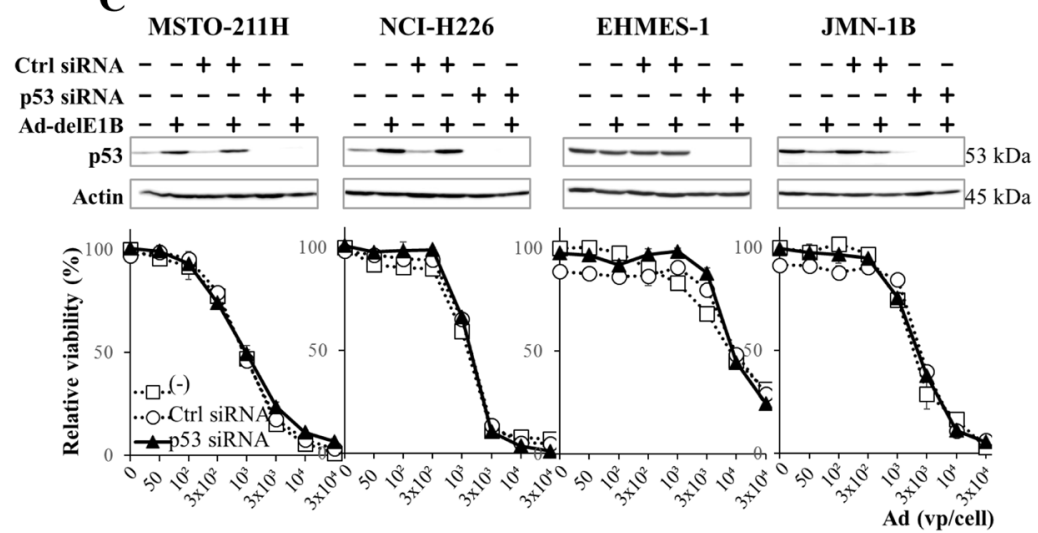

Fig. 1 Ad-delE1B-mediated growth inhibitory effects were independent of p53 expression. A Growth inhibitory effects of Ad-delE1B. Mesothelioma with the wild-type $p 53(\mathrm{MSTO}-211 \mathrm{H}, \mathrm{NCl}-\mathrm{H} 226$ and $\mathrm{NCl}-\mathrm{H} 28$ ) or mutated $p 53$ genotype (EHMES-1 and JMN-1B) were treated with Ad-delE1B or Ad-LacZ at various vp for 4 days. The cell viabilities were measured with the WST agent and relative viability was calculated based on untreated cells. IC 50 values (vp/cell) were calculated with GraphPad Prism software. Averages and SE bars are shown ( $n=3$ ). B Cells were treated with Ad-delE1B $\left(3 \times 10^{3} \mathrm{vp} /\right.$ cell) and cultured for 2 or 4 days. Expression of p53, phosphorylated p53 at Ser 15 (p-P53 S15), and actin as a control was examined with Western blot analysis. C Cells transfected with p53 siRNA or with non-target control siRNA (Ctrl siRNA) were treated with Ad-delE1B $\left(3 \times 10^{3} \mathrm{vp} /\right.$ cell) for 4 days. Expression of p53 and actin as a control was examined with Western blot analysis. Cells transfected with siRNA were also treated with Ad-delE1B at various vp for 4 days and the cell viabilities were measured with the WST agent. Relative viability was calculated based on untreated cells. Averages and SE bars are shown $(n=3)$.

lower than those to mutated $p 53$ cells $(30.85 \pm 2.76)(p<0.01)$. Likewise, the values of RG7112 in wild-type cells $(2.55 \pm 0.43)$ were less than those in mutated cells $(10.51 \pm 0.49)(p<0.01)$. We then examined the expression of p53 and the phosphorylation in cells treated with MDM2 inhibitors (Fig. 2B). Wild-type p53 cells augmented p53 and the phosphorylation levels in a dosedependent manner. We observed similar changes in JMN-1B cells despite being insensitive the inhibitors. EHMES-1 cells did not show increase of p53 or the phosphorylation. We further examined a role of $\mathrm{p} 53$ in the MDM2 inhibitors-induced cytotoxicity by knocking down p53 (Fig. 2C). All the wild-type p53 cells became insensitive to nutlin-3a or RG7112 after p53 siRNA treatments, whereas cells treated with control siRNA remained sensitive. These data indicated that MDM2 inhibitors produced cytotoxicity in a p53-dependent manner through increased endogenous p53 levels.

\section{Ad-delE1B and MDM2 inhibitors produced combinatory effects}

We investigated a role of upregulated p53 in Ad-delE1B-mediated cytotoxicity with MDM2 inhibitors. We treated Ad-delE1B-infected cells with the inhibitors and examined growth inhibition (Fig. 3A).
Combination of Ad-delE1B and nutlin-3a or RG7112 showed $\mathrm{Cl}$ values less than 1 at Fa points between 0.2 and 0.8 (nutlin-3a) or between 0.2 and 0.7 (RG7112) in MSTO-211H and $\mathrm{NCl}-\mathrm{H} 226$ cells, demonstrating that the combination produced synergistic effects. A combinatory use of Ad-delE1B and MDM2 inhibitors however produced rather antagonistic effects in EHMES-1 and JMN-1B cells because the $\mathrm{Cl}$ values were above 1 (Fig. 3B). We also examined the combinatory effects on cell survivals with a dye exclusion assay (Fig. 3C). Ad-delE1B or MDM2 inhibitors alone produced cytotoxicity in wild-type $p 53$ mesothelioma and the combination achieved cytotoxic effects greater than a single treatment. In contrast, EHMES-1 and JMN-1B cell numbers decreased with AddelE1B at $48 \mathrm{~h}$ but no further inhibition was detected in the combination. These results suggested that combination of $\mathrm{Ad}$ delE1B and MDM2 inhibitors achieved growth inhibition in a p53dependent manner.

\section{Augmented apoptosis by the combination}

We examined cell cycle progression and apoptosis induction of the wild-type p53 cells treated with the combination (Fig. 3D, Supplementary Table 2). MDM2 inhibitors decreased S-phase populations and Ad-delE1B induced hyperploidy (over $4 \mathrm{~N}$ ) fractions 

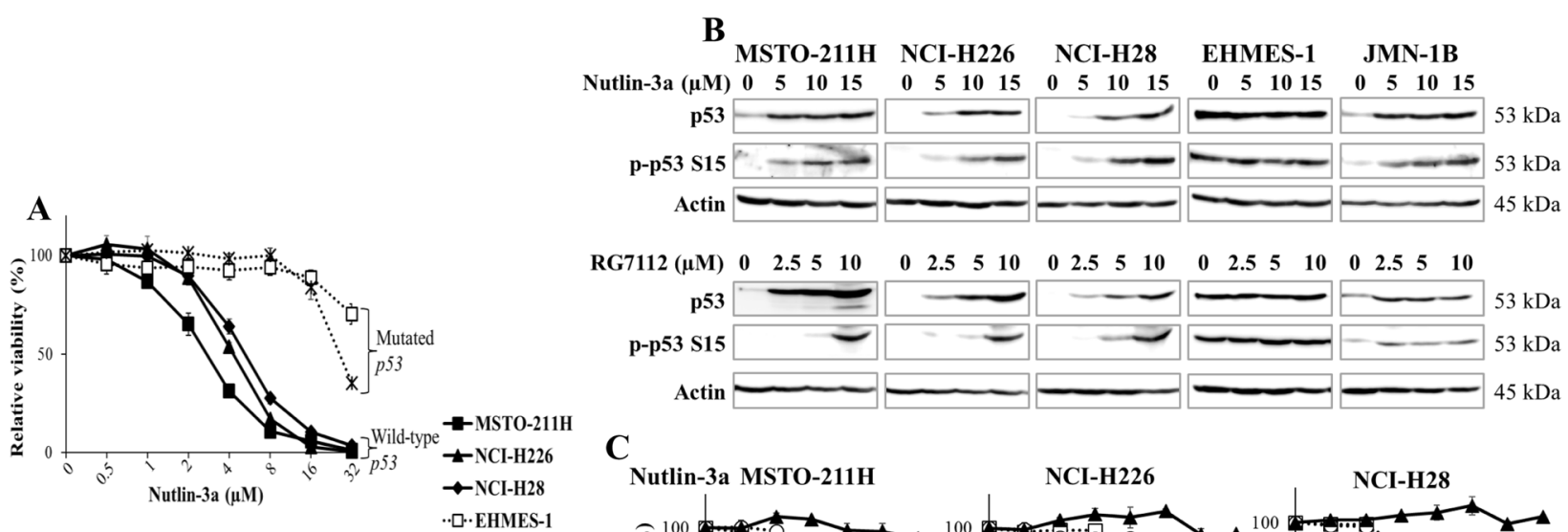

C
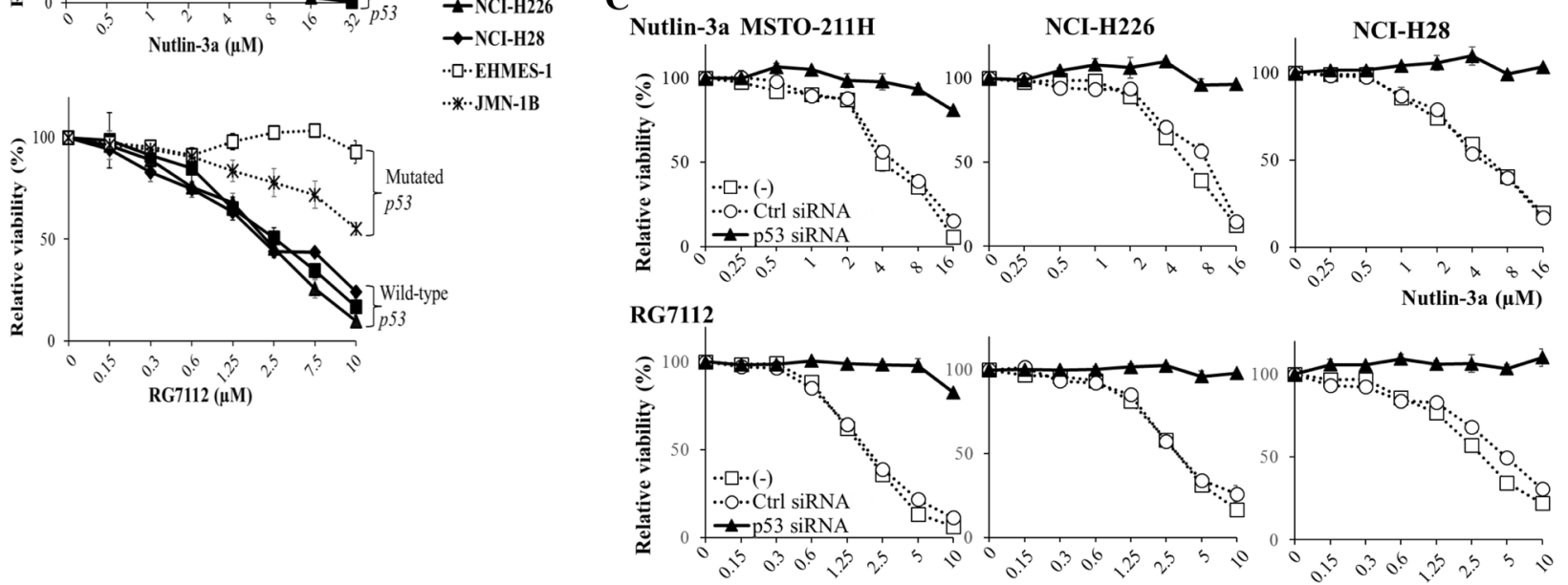

RG7112 ( $\mu$ M)

Fig. 2 MDM2 inhibitors increased p53 expression in wild-type p53 mesothelioma and the growth inhibition was relevant to p53 genotype. A Cells were treated with various concentrations of nutlin-3a for 4 days or RG7112 for 3 days. The cell viabilities were measured with the WST agent and relative viability was calculated based on untreated cells. Averages and SE bars are shown $(n=3)$. B Cells were treated with various concentrations of nutlin-3a or RG7112 as indicated for 2 days. Expression of p53 and phosphorylated p53 at Ser 15, and actin as a control was examined with western blot analysis. C Cells transfected with p53 siRNA or control siRNA were treated with various concentrations of nutlin-3a or RG7112 for 3 days and the cell viabilities were measured with the WST agent. Relative viability was calculated based on untreated cells. Averages and SE bars are shown $(n=3)$.

in MSTO-211H cells. The combination increased sub-G1 and hyperplioidy compared with cells treated with Ad-delE1B alone. Likewise, MDM2 inhibitors decreased S-phase populations, and AddelE1B increased sub-G1 and hyperploidy fractions in $\mathrm{NCl}-\mathrm{H} 226$ cells which constantly showed a different peak in a sub-G1 region from cell death-associated sub-G1 fractions. The combination increased sub-G1 populations greater than the single treatment and augmented hyperploidy. In contrast, Ad-LacZ did not influence cell cycle and combination of Ad-LacZ and the inhibitors showed similar cell cycle distributions as the inhibitor alone-treated case. These data indicated that MDM2 inhibitors enhanced Ad-delE1Bmediated effects.

We also examined apoptosis induction with the annexin $\mathrm{V}$ staining (Fig. 3E). An annexin V-positive fraction increased in cells infected with Ad-delE1B or treated with MDM2 inhibitors. The combination expanded the population greater than the single treatment in MSTO- $211 \mathrm{H}$ and $\mathrm{NCl}-\mathrm{H} 226$ cells. These data showed that the combination augmented apoptotic cell death.

\section{Combinations augmented p53 and caspase cleavages}

We investigated the expression of p53 and the related molecules in wild-type $p 53$ cells treated with the combination (Fig. 4A). Cells treated with Ad-delE1B, nutlin-3a, or RG7112 showed increased p53 levels and the phosphorylation, and those with the combination further augmented the expression. Ad-delE1B induced cleavage of caspase- 3 and PARP, and the combination further induced the cleavages. The combination increased caspase-8 cleavage caused by Ad-delE1B although expression of Fas and FADD was not enhanced in the combination in MSTO$211 \mathrm{H}$ cells. In contrast, $\mathrm{NCl}-\mathrm{H} 226$ cells showed differential expression levels of Fas and FADD in the combination. Fas expression was upregulated in $\mathrm{NCl}-226 \mathrm{H}$ cells treated with $\mathrm{Ad}-$ delE1B or the inhibitors, and the FADD increased in those with AddelE1B. Combination with RG7112 enhanced Fas and FADD expression, whereas the combination with nultin-3a rather decreased the levels. Ad-delE1B and the inhibitors stimulated cleavage of caspase- 9 at $35 \mathrm{kDa}$ molecules, and the combination further augmented the cleavages in both cells. Expression of PUMA and Bax increased in MSTO- $211 \mathrm{H}$ cells treated with either nutlin-3a or RG7112 but decreased in those with the combination. In contrast, the levels in $\mathrm{NCl}-226 \mathrm{H}$ cells treated with the combination were greater than those treated with a single agent. Transduction with Ad-LacZ scarcely influenced these expressions even in the combination except increased PUMA levels in nutlin3a-treated MSTO- $211 \mathrm{H}$ cells. Expression of p53 and the phosphorylation remained unchanged in EHMES-1 cells and was not augmented in JMN-1B cells treated with the combination (Fig. 4B). These data suggested that the combination augmented p53 levels and activated apoptotic pathways in wild-type p53 mesothelioma but activation of the extrinsic or intrinsic pathways was dependent on cells tested.

We also examined whether autophagy pathways were relevant to cell death (Fig. 4C). Expression of Atg-5, Beclin-1, or transition of LC3A/B I to LC3A/B II was scarcely influenced by Ad-delE1B or MDM2 inhibitor, and was not enhanced by the combination. 


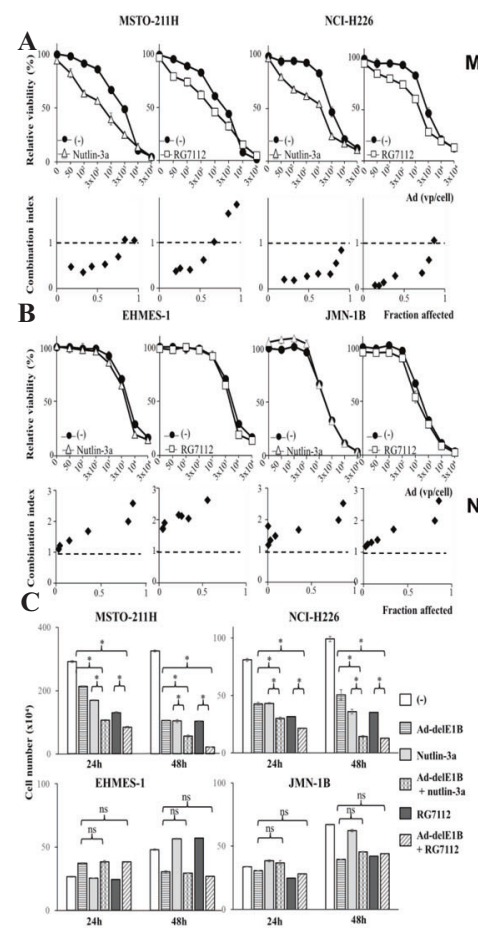

D
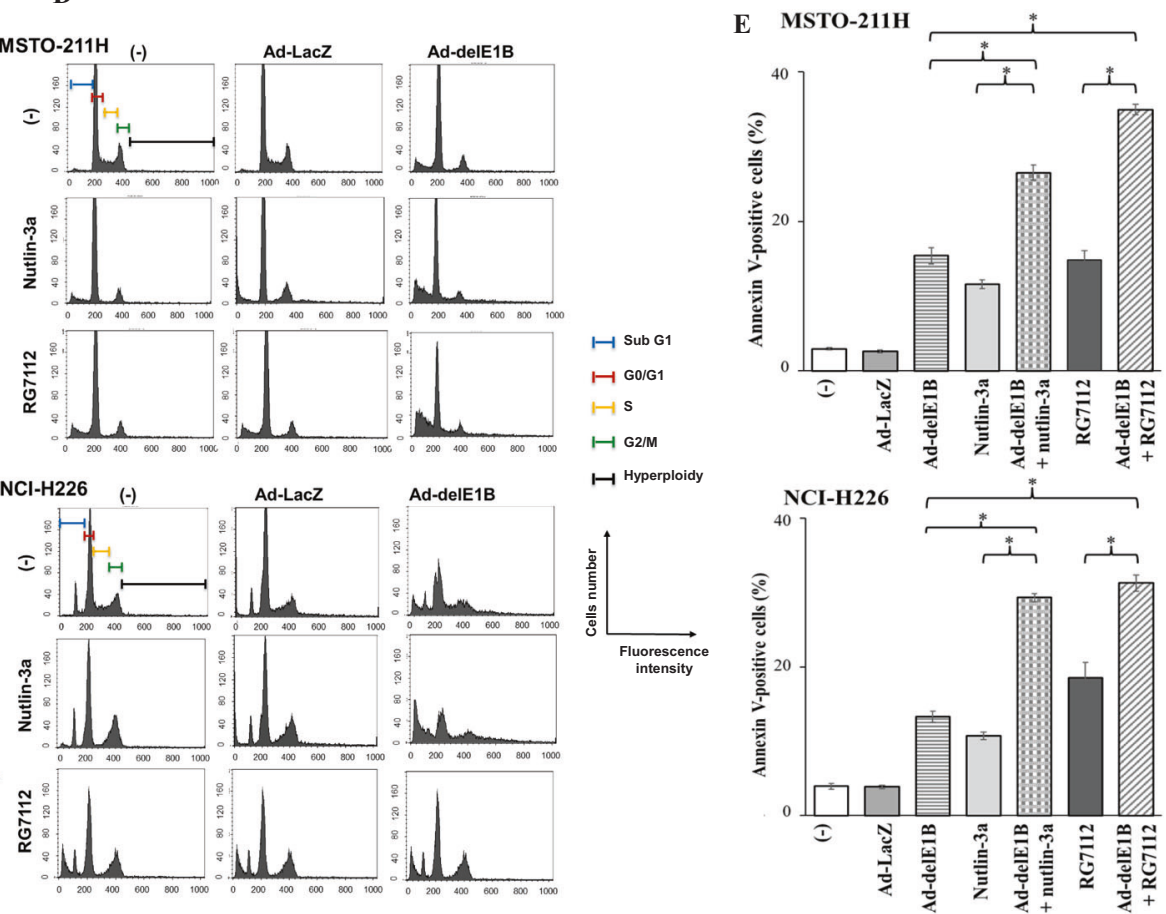

Fig. 3 MDM2 inhibitors produced synergistic growth inhibitory effects and changed cell cycle progression with Ad-delE1B on wild-type p53 cells. A Wild-type $p 53$ or B mutated $p 53$ cells were infected with various vp of Ad-delE1B for 2 days, and then treated with nutlin-3a (MSTO-211H: $0.5 \mu \mathrm{M}, \mathrm{NCl}-\mathrm{H} 226$, EHMES-1, and JMN-1B: $1 \mu \mathrm{M})$ or RG7112 $(0.15 \mu \mathrm{M})$ for further 2 days. The cell viabilities were measured with the WST agent. Relative viability was calculated based on untreated cells. Averages and SE bars are shown $(n=3)$. Cl values were plotted at respective Fa points. $\mathrm{Cl}<1, \mathrm{Cl}=1$ and $\mathrm{Cl}>1$ indicate synergistic, additive, and antagonistic actions, respectively. $\mathrm{C}$ Cells were infected with AddelE1B (MSTO-211 H and NCI-H226: $1.5 \times 10^{3} \mathrm{vp} /$ cell, EHMES-1 and JMN-1B: $3 \times 10^{3} \mathrm{vp} / \mathrm{cell}$ ) for 2 days, and then treated with nutlin-3a (MSTO$211 \mathrm{H}$ and NCl-H226: $5 \mu \mathrm{M}$, EHMES-1, and JMN-1B: $15 \mu \mathrm{M}$ ) or RG7112 (MSTO-211H and NCI-H226: $3 \mu \mathrm{M}$, EHMES-1 and JMN-1B: $10 \mu \mathrm{M}$ ). Live cells numbers were then counted on 24 and $48 \mathrm{~h}$ later. ns not significant, ${ }^{*} p<0.001$ (GraphPad Prism 6, La Jolla). D Cells were infected with AddelE1B or Ad-LacZ $\left(3 \times 10^{3} \mathrm{vp} /\right.$ cell) for 2 days, and then treated with nutlin-3a $(5 \mu \mathrm{M})$ or RG7112 $(5 \mu \mathrm{M})$. Cell cycle distribution was determined with flow cytometry. Representative histogram of MSTO-211H cells at $36 \mathrm{~h}$ after treatment with the inhibitor and that of $\mathrm{NCl}-\mathrm{H} 226 \mathrm{at} 48 \mathrm{~h}$. E Percentages of annexin V-positive cells. Cells were treated as above and annexin V-positive cells were determined with flow cytometry in MSTO- $211 \mathrm{H}$ cells at $36 \mathrm{~h}$ after the inhibitor treatment and in $\mathrm{NCl}-\mathrm{H} 226$ at $48 \mathrm{~h}$. Data are presented as averages with SE bars $(n=3)$. ${ }^{*} p<0.01$.

These data indicated that autophagy was not induced in wild-type p53 cells.

\section{Combination activated DNA damage signals}

We examined DNA damage responses induced by Ad-delE1B and the combination (Fig. 4D). Expression of $\mathrm{\gamma}-\mathrm{H} 2 \mathrm{AX}$ increased with Ad-delE1B and to a lesser extent with MDM2 inhibitors, and the level was further augmented in the combination. Phosphorylated KAP1 at Ser 824, a different DNA damage marker, was induced by Ad-delE1B and RG7112, and was further elevated in the combination. We also analyzed the expression of molecules in ATM-Chk2 and ATR-Chk1 pathways which were relevant to H2AX and KAP1 phosphorylation. Expression levels of ATM, Chk1 and Chk2 were constant among cells treated with Ad-delE1B and/or MDM2 inhibitors, but phosphorylation of ATM at Ser 1981 and Chk2 at Thr 348 was augmented in MSTO-211H cells treated with combination of Ad-delE1B and RG7112. Ad-delE1B, nutlin-3a or RG7112 alone increased the phosphorylation of ATM and Chk2 in $\mathrm{NCl}-\mathrm{H} 226$ cells, and the combination further stimulated the phosphorylation. ATR expression remained unchanged in MSTO$211 \mathrm{H}$ cells but that in $\mathrm{NCl}-\mathrm{H} 226$ cells was down-regulated by $\mathrm{Ad}$ infections and the combination. Phosphorylation levels of ATR at Ser 428 and Chk1 at Ser 345 remained unchanged in cells treated with the agent alone or the combination except elevated Chk1 phosphorylation in $\mathrm{NCl}-\mathrm{H} 226$ cells treated with nutlin-3a or RG7112. These results indicated that Ad-delE1B in combination with MDM2 inhibitors-induced DNA damage mainly through activation of the ATM-Chk2 rather than the ATR-Chk1 pathway.

\section{MDM2 inhibitors augmented Ad-delE1B replications and suppressed tumor growth in vivo}

We investigated a role of MDM2 inhibitors in production of viral progenies (Fig. 5). Ad-delE1B-infected cells expressed E1A, a product of the immediate early gene, and the expression was upregulated in combination of MDM2 inhibitors (Fig. 5A). We estimated the progeny numbers with the $\mathrm{TCID}_{50}$ method and found that both inhibitors enhanced the production in the wildtype $p 53$ cells. In contrast, mutated p53 mesothelioma did not increase the production with MDM2 inhibitors and the E1A expression remained unchanged (Fig. 5B). We further examined an involvement of $\mathrm{p} 53$ in enhancing viral replications by knocking down p53 (Fig. 5C). Cells treated with p53 siRNA downregulated Ad-delE1B-induced p53 levels and minimally augmented the levels with MDM2 inhibitors. Ad-delE1B-induced EIA was not influenced by the siRNA treatment but the MDM2 inhibitorsmediated E1A upregulation decreased. A titration of viral productions indicated that enhanced Ad-delE1B replications by the inhibitors were eliminated with the p53 siRNA and the production was indifferent between cells infected with Ad-delE1B and those treated with the combination. These data demonstrated that an increased p53 level by MDM2 inhibitors facilitated replications of Ad-delE1B.

We next evaluated anti-tumor effects of the combination in a xenograft model (Fig. 5D). An administration of Ad-delE1B, nutlin$3 a$, or RG7112 alone inhibited tumor growth and the combination further reduced the tumor weights. No significant weight loss was observed in mice treated with the combination. These in vivo data 


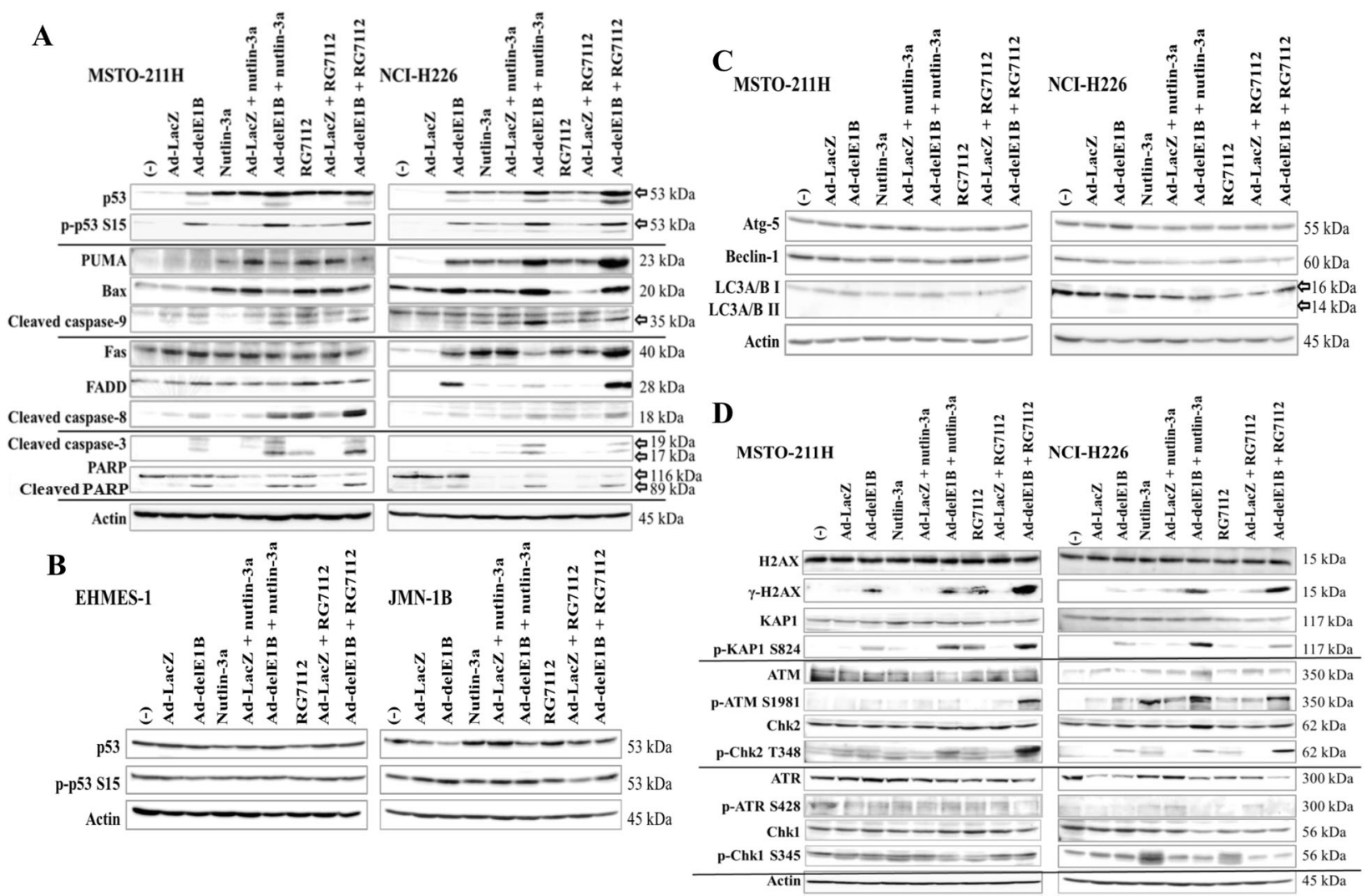

Fig. 4 Combination of Ad-delE1B and MDM2 inhibitors augmented apoptotic pathways and DNA damage signals. A Cells were infected with Ad-delE1B or Ad-LacZ $\left(3 \times 10^{3} \mathrm{vp} / \mathrm{cell}\right)$ for 2 days, and then treated with nutlin-3a (15 $\left.\mu \mathrm{M}\right)$ or RG7112 (10 $\left.\mu \mathrm{M}\right)$ for further $16 \mathrm{~h}$ in MSTO$211 \mathrm{H}$ or $36 \mathrm{~h}$ in $\mathrm{NCl}-\mathrm{H} 226$. Cell lysate of wild-type (A) or mutated $p 53$ cells (B) were subjected to western blot analysis and expression levels of molecules associated with p53 (A, B), apoptosis (A), autophagy (C), and DNA damage pathways (D) were examined with respective Ab as indicated.

showed that the combination produced combinatory anti-tumor effects on mesothelioma developed in the pleura cavity. We also confirmed that Ad-delE1B-mediated E1A expression of tumors was upregulated by a combinatory use of MDM2 inhibitors (Supplementary Fig. 4).

\section{Enhanced NFI expression by MDM2 inhibitors}

We analyzed the expression of four major transcriptional factors, B23, TFIID, Oct1, and NFI, involved in Ad replications (Fig. 6A). Expression of B23 was not influenced by Ad-delE1B, MDM2 inhibitors, or the combinations. TFIID were augmented in MSTO$211 \mathrm{H}$ cells treated with Ad-delE1B and the combination with RG7112, but remained unchanged in $\mathrm{NCl}-\mathrm{H} 226$ cells. Oct1 expression was downregulated in $\mathrm{NCl}-\mathrm{H} 226$ cells treated with RG7112 and combination of Ad-delE1B and RG7112, but was not affected in MSTO- $211 \mathrm{H}$ cells. In contrast, NFI expression increased with Ad-delE1B infections, and was further augmented in the combination with nutlin-3a in $\mathrm{NCl}-\mathrm{H} 226$ cells. The combination with RG7112 also enhanced NFI expression in both cells. We also examined the upregulated NFI levels with immunostaining data (Fig. 6B). MSTO-211H and $\mathrm{NCl}-\mathrm{H} 226$ cells infected with Ad-delE1B but not Ad-LacZ-induced NFI, but those treated with nutlin-3a or RG7112 remained negative for NFI. Combination of Ad-delE1B and the inhibitors strongly augmented NFI expression in the nucleus and cytoplasm. These data collectively suggested that Ad-delE1Binduced NFI expression was mediated by upregulated p53 expression.

We then investigated a role of p53 in NFI expression with siRNA (Fig. 6C) and replication-deficient Ad expressing wild-type p53 (Ad-p53) (Fig. 6D). MSTO-211H and $\mathrm{NCl}-\mathrm{H} 226$ cells treated with p53 siRNA did not express NFI when they were infected with Ad-
delE1B or treated with the combinatory use of nutlin-3a. Transduction of Ad-p53 augmented p53 levels and induced NFI expression according to Ad amounts. We noticed that Ad-p53 mainly induced NFI in nuclei in contrast to cells treated with the combination. These data indicated that induction of NFI was associated with p53 expression and NFI was one of the p53 target molecules.

\section{DISCUSSION}

We investigated a role of p53 in cytotoxicity induced by $\mathrm{p} 53-$ activating replication-competent $\mathrm{Ad}$ and demonstrated that increase of endogenous p53 with MDM2 inhibitors augmented Ad-induced DNA damage responses and promoted the viral replications through augmented NFI expression. Ad-delE1B upregulated $\mathrm{p} 53$ levels in the wild-type $p 53$ mesothelioma but the p53 upregulation did not contribute to the Ad-mediated cytotoxicity. Nevertheless, further augmentation of p53 levels with an MDM2 inhibitor produced synergistic cytotoxicity in combination with Ad-delE1B. In addition, the present study firstly demonstrated to our knowledge that the p53 level played a vital role in $\mathrm{NFI}$ induction and subsequent production of $\mathrm{Ad}$ progenies.

Ad-delE1B preferentially replicated in tumors and immortalized cells, but not in normal cells (Supplementary Fig. 1). The replications were irrelevant to the $p 53$ genotype of infected cells [14]. We previously reported that Ad-delE1B increased p53 levels and induced apoptosis in wild-type p53 mesothelioma with CDKN2A deletion, and suggested a role of p53 in Ad-delE1Binduced cytotoxicity [11]. Moreover, forced expression of p14 impaired the Ad-delE1B-mediated cytotoxicity [15] and 
A

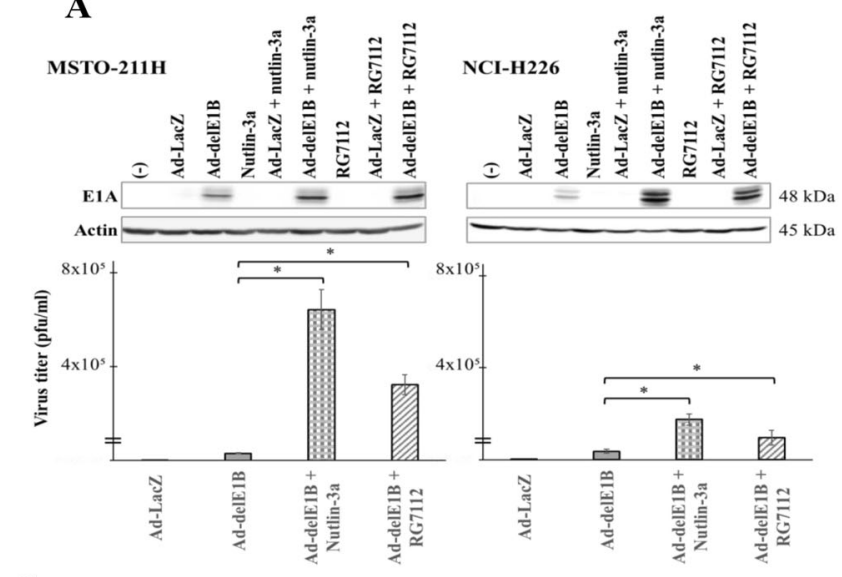

C

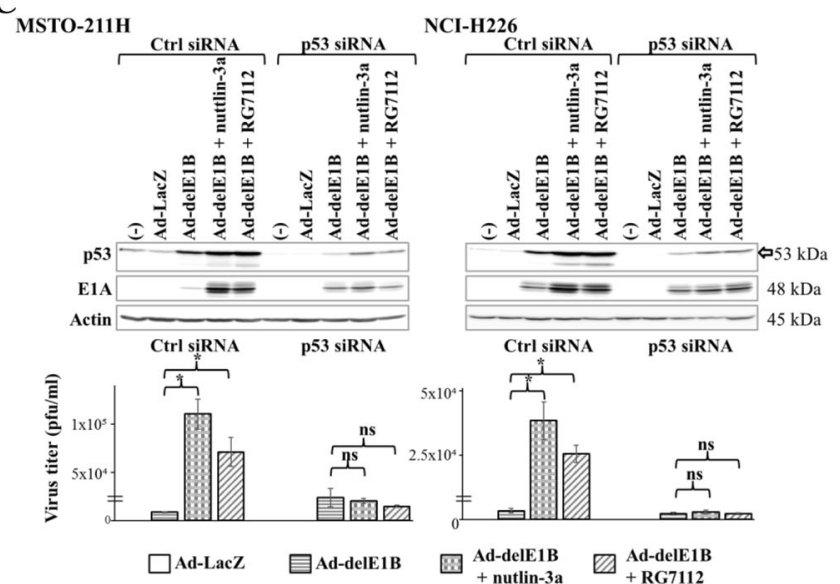

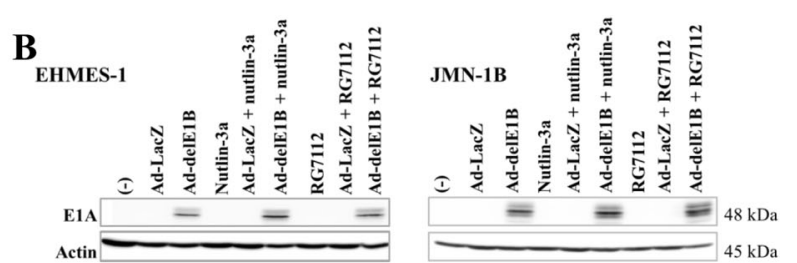

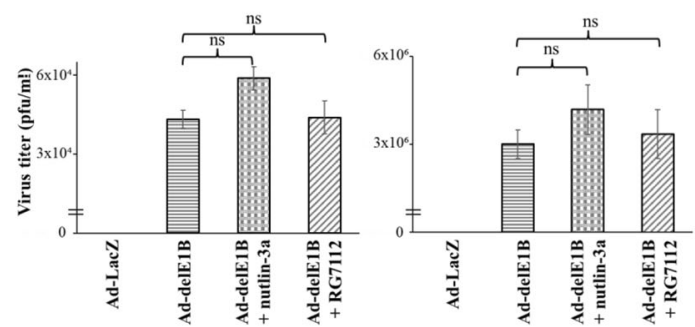

D

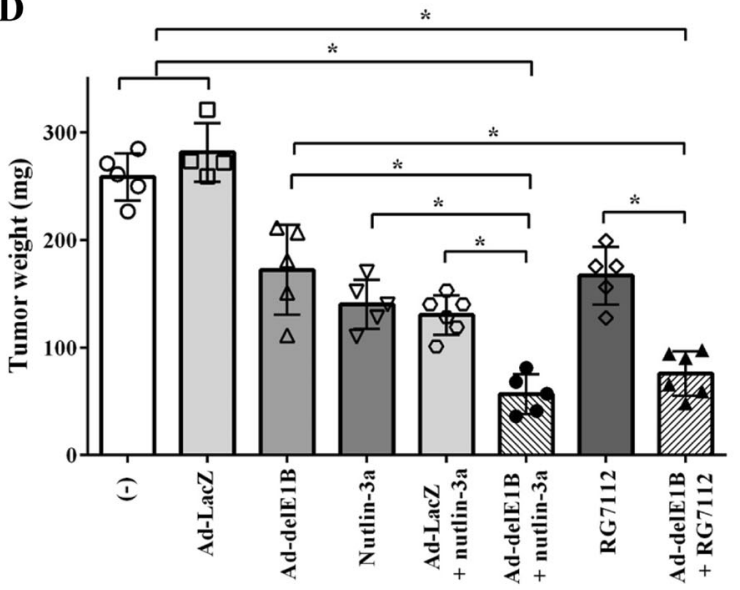

Fig. 5 MDM2 inhibitors augmented production of Ad-delE1B progenies through p53 expression and combination suppressed tumor growth in vivo. Wild-type $(\mathbf{A}, \mathbf{C})$ and mutated $p 53$ cells $(\mathbf{B})$ were infected with Ad-delE1B or Ad-LacZ ( $\left.3 \times 10^{3} \mathrm{vp} / \mathrm{cell}\right)$ for 2 days, and treated with nutlin-3a $(15 \mu \mathrm{M})$ or RG7112 $(10 \mu \mathrm{M})$ for further $16 \mathrm{~h}$ in MSTO-211 H or $36 \mathrm{~h}$ in NCl-H226. Expression of p53, E1A, and actin as a control was examined with Western blot analysis. Quantitation of virus progenies was examined with the TCID ${ }_{50}$ method. Averages and SE bars are shown $(n=3)$. ns not significant, ${ }^{*} p<0.01$. C Expression of p53 was knocked down with p53 siRNA and control siRNA was used as a control. Cells transfected with siRNA were infected with Ad-delE1B for 2 days, and then treated with nutlin-3a (15 $\mu \mathrm{M})$ or RG7112 (10 $\mu \mathrm{M})$ for further $16 \mathrm{~h}$ in MSTO- $211 \mathrm{H}$ or $36 \mathrm{~h}$ in NCl-H226. D Ad-delE1B $\left(1 \times 10^{10} \mathrm{vp} / \mathrm{mouse}\right)$ and MDM2 inhibitors $(1 \mathrm{mg})$ produced combinatory anti-tumor effects in an animal experiment. Ad-LacZ was used as a control. The data indicated individual tumor weights and an interquartile range of weights of tumors. ${ }^{*} p<0.01$.

consequently MDM2 activity was also involved in the cytotoxicity. The current study further demonstrated that the cytotoxicity was not linked with p53 expression and combination with MDM2 inhibitors increased the Ad-delE1B cytotoxicity. These data therefore suggested a putative threshold level of p53 as for Ad-mediated cytotoxicity. Previous studies investigated a role of p53 in cytotoxicity of replication-competent $\mathrm{Ad}$ and demonstrated that p53 enhanced the cytotoxicity [16]. The Ad used in these studies were different in the structures from Ad-delE1B, for example, expressing mutated p53 which was degradation-resistant but functional in the transcriptional activity [17], or having an expression unit of wild-type p53 [18, 19]. A possible mechanism for the enhanced cytotoxicity was not well analyzed in these studies but several mechanisms were postulated depending on $\mathrm{Ad}$ structures, which included autophagy induction [18], microRNAinduced p21 inhibition [19], and p53-mediated apoptosis [6]. Viral replications examined in these reports were scarcely influenced or rather decreased due to facilitated death of viruses-producing cells $[6,9,11]$. Furthermore, they used tumors in which the p53 downstream pathways were disturbed $[6,18]$. We presumed that NFI was not well induced in these $\mathrm{p} 53$ non-functional cells and a non-NFI mechanism was consequently involved in such cases. A possible $\mathrm{p} 53$ role in cytotoxicity of replication-competent Ad can be influenced by cellular factors, which achieve different outcomes as for viral progeny productions.

We demonstrated that nutlin-3a and RG7112 increased endogenous p53 levels in combination with Ad-delE1B and induced cleavages of caspase- $3,-8$ and -9 , and PARP. The combination however differentially influenced expression levels of Fas, FADD, Bax, and PUMA. Expression of Fas and FADD for example remained unchanged in MSTO-211H cells treated with Ad-delE1B and nutlin3a or RG7112, whereas that in $\mathrm{NCl}-\mathrm{H} 226$ cells was differently affected. These data indicated that upregulated p53 levels activated caspase cleavages in wild-type p53 cells but detailed mechanisms of apoptosis induction were not identical among cells tested. Nevertheless, the combination invariably augmented AddelE1B-induced DNA damage responses, which were evidenced by increased $\mathrm{y}-\mathrm{H} 2 \mathrm{AX}$ and phosphorylated KAP1 levels. ATM and Chk2 were involved in the DNA damage induced by Ad-delE1B [20] and we demonstrated that an MDM2 inhibitor enhanced the ATMChk2-mediated DNA damage, but the ATR-Chk1 pathway was scarcely involved. The differential DNA damage responses suggested that Ad-delE1B replications induced double-strand DNA breaks and MDM2 inhibitors preferentially supported ATM-Chk2 activation which was linked with p53 phosphorylation. KAP1 was phosphorylated by Ad replications as well as a non-viral 


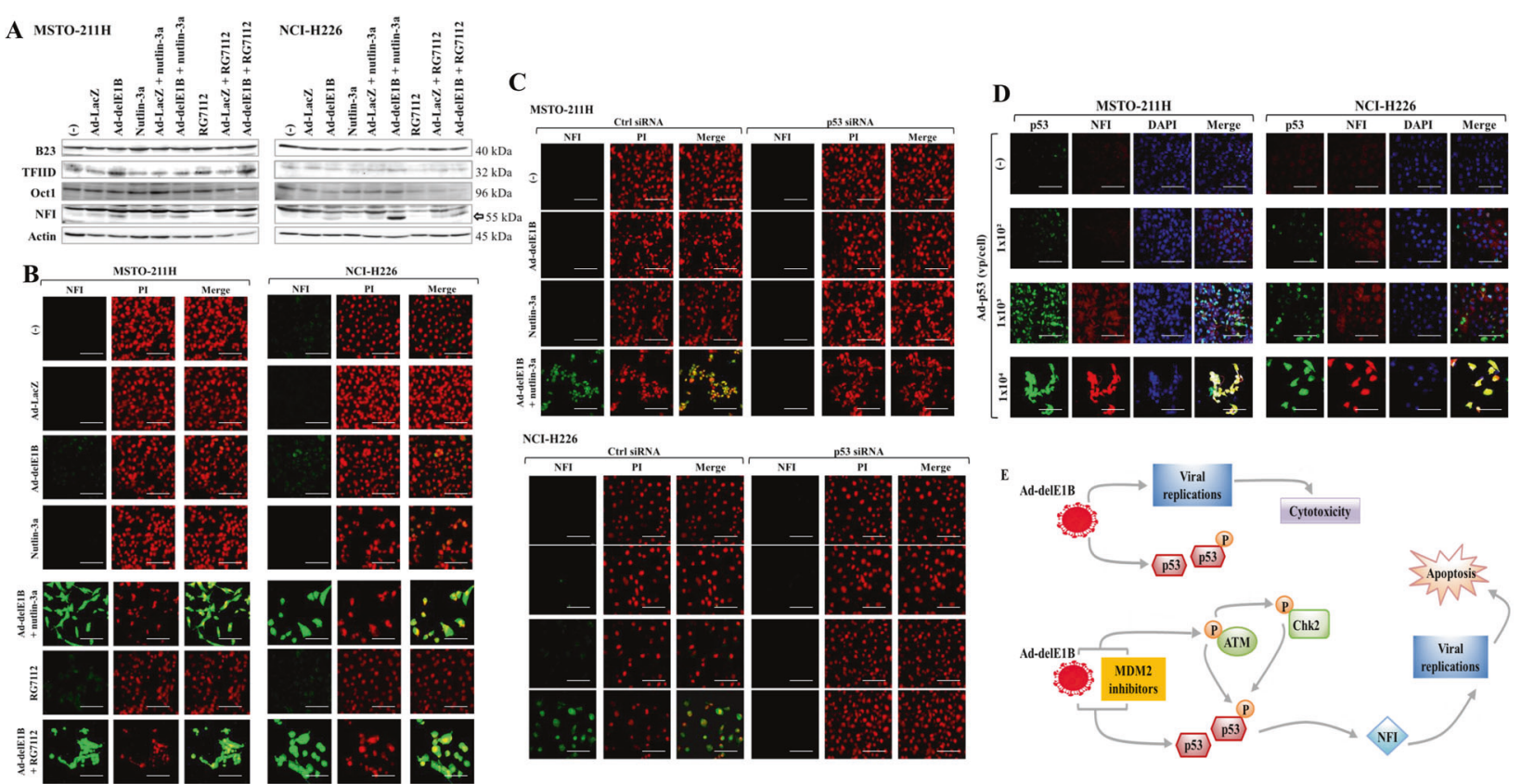

Fig. 6 Enhanced NFI expression with MDM2 inhibitors. Cells were infected with Ad-delE1B or Ad-LacZ ( $\left.3 \times 10^{3} \mathrm{vp} / \mathrm{cell}\right)$ for 2 days, and then treated with nutlin-3a $(15 \mu \mathrm{M})$ or RG7112 $(10 \mu \mathrm{M})$ for further $16 \mathrm{~h}$ in MSTO-211H or $36 \mathrm{~h}$ in NCl-H226. A Cell lysate was examined for expression of cellular factors linked with Ad replications with Western blot analysis. NFI: An upper band ubiquitously expressed was non-specific and a lower band corresponding to $55 \mathrm{kDa}$ was authentic. B-D Cells were stained with Ab against NFI or p53, or with DAPI or PI, and the images were analyzed with a laser confocal microscope. C Expression of p53 was knocked down with p53 siRNA and non-target siRNA was used as a control. Cells transfected with siRNA were infected with Ad-delE1B for 2 days, and then treated with nutlin-3a (15 $\mu \mathrm{M})$ or RG7112 (10 $\mu \mathrm{M})$ for further $16 \mathrm{~h}$ in MSTO-211 $\mathrm{H}$ or $36 \mathrm{~h}$ in $\mathrm{NCl}-\mathrm{H} 226$. D Cells were infected with several vp of Ad-p53 as indicated for 2 days. E A model of AddelE1B and p53 upregulated by MDM2 inhibitors in Ad-mediated cytotoxicity and virus replications. Scale bar: $10 \mu \mathrm{m}$.

mechanism, and the phosphorylation was further upregulated by E1B55kDa deletion since KAP1 bound to E1B55kDa molecules $[20,21]$. KAP1 is in fact a negative regulator for Ad replications but the phosphorylation of KAP1 promoted decondensation of cellular and viral genome, which resulted in transcriptional activation of the genes required for Ad replications. The increased KAP1 phosphorylation by MDM2 inhibitors was therefore attributable to enhanced DNA damage induced by viral replications and also contributed to production of viral progenies. We also noticed increased hyperploidy fractions induced by Ad-delE1 and the combination with MDM2 inhibitors. We previously showed that the hyperploidy was not linked with aberrant chromosomal numbers or abnormal cell divisions [11] and speculated that the hyperploidy may be partly associated with increased nuclear DNA contents caused by Ad replications.

We demonstrated that MDM2 inhibitors enhanced viral production in the wild-type $p 53$ cells and knocking down of p53 decreased the progeny production to the level of Ad-infected cells, which indicated that p53 levels regulated the viral replications. The viral production in normal cells with intact CDKN2A region is interesting but Ad-delE1B were less cytotoxic (Supplementary Fig. 1) and MDM2 inhibitors were only toxic at a high concentration in normal cells. A possible effect of the combination on normal tissues can be examined in vivo but we did not observe body weight loss in the animal study (data not shown). The current study also examined cellular factors involved in Ad replications and showed that NFI expression was slightly induced by Ad-delE1B and further upregulated by MDM2 inhibitors [22]. Knocking down of p53 depleted the NFI upregulation, and transduction with Ad-p53 increased the expression in a dose-dependent manner. These data indicated that the NFI expression level was regulated by $\mathrm{p} 53$. Induction of NFI in AddelE1B-infected cells was therefore attributable to increased endogenous $\mathrm{p} 53$, but we cannot rule out a possible induction of $\mathrm{NFI}$ by $\mathrm{Ad}$ replications. A compared use of Ad-delE1B and replication-competent $\mathrm{Ad}$ bearing p53-inactivating E1B55kDa molecules can differentiate contribution of $\mathrm{p} 53$ and viral replications to NFI induction. We also tested a role of NFI in the viral production by knocking down of NFI with siRNA and demonstrated that NFI regulated the production (Supplementary Fig. 2). We noticed that an expression level of NFI may not be directly associated with the production of the viral progenies because the production in MSTO- $211 \mathrm{H}$ cells was greater than that in $\mathrm{NCl}-226 \mathrm{H}$ cells. These data suggested that the viral replications were influenced by many cellular factors including synthesis of viral late proteins and the viral packaging activities.

The present study firstly reported that p53 augmented NFI levels and demonstrated a possible p53-mediated upregulation of NFI. A previous study however showed p53-induced NF1 downregulation in hepatocellular carcinoma [23]. Moreover, NFI augmented p53 expression in mammary glands [24] and decreased the expression in glioblastoma [25]. These data therefore suggested that a reciprocal regulation between $\mathrm{p} 53$ and $\mathrm{NFI}$ and the regulation was dependent on cells used. We then examined the regulation by knocking down of NFI with siRNA in wild-type $p 53$ mesothelioma (Supplementary Fig. 3). We found that decreased NFI expression downregulated p53 levels, indicating that NFI was a positive regulator for $\mathrm{p} 53$. NFI is a transcriptional factor and regulates a number of genes [26], but how NFI controls the $p 53$ gene remains unclear. A putative binding site of $\mathrm{NFI}$ or $\mathrm{p} 53$ in the regulatory region of respective genes was not identified and consequently mechanism of the reciprocal regulation between NFI and p53 remains unknown. NFI showed a tumor-promoting activity through facilitating cell proliferations and migration [25], and consequently elevated NFI expression may be linked with preferential cytotoxicity of oncolytic Ad in tumors compared with normal tissues. A functional role of p53 and NFI in Ad-delE1B-mediated cytotoxicity can be different from that in other types of replication-competent Ad types bearing E1B55kDa molecules. E1B55kDa mediated KAP1 dephosphorylation and p53 degradation, and influenced a number 
of signal pathways $[20,27]$. A possible axis of ATM-Chk2-p53-NFI demonstrated in the present study can be restricted in the AddelE1B-induced cytotoxicity. Moreover, NFI induction can be subjected to a putative p53 threshold level since Ad-delE1B minimally induced NFI expression in contrast to the significant induction in the combination with MDM2 inhibitors. On the other hand, we detected a dose-dependent NFI induction with Ad-p53, suggesting that the NFI level was correlated with p53 expression when the p53 level increased beyond the threshold.

We demonstrated a clinical feasibility of the combination in an in vivo experimental model. Ad-delE1B is clinically used in China [28] and p53-activating agents were examined for the efficacy in a clinical setting $[29,30]$. Mesothelioma increased p53 levels through inhibition of MDM2 and DNA damage, and consequently activated p53 downstream pathway and cell death induction. These data suggest that p53 stimulation is a target of mesothelioma treatments. An injection of Ad vectors into the pleural cavity is technically easy and several clinical studies demonstrated that intrapleural administration of Ad was safe [31]. A clinical trial of the combination can reveal therapeutic efficacy and possible adverse effects on normal tissues as well.

In conclusions, we showed that increase of p53 levels by AddelE1B infection did not contribute to the Ad-mediated cytotoxicity, but further enhanced p53 expression with MDM2 inhibitors was associated with activation of the ATM-Chk2 pathway and augmented the cytotoxicity through upregulated $\mathrm{NFI}$ expression (summarized in Fig. 6E). A majority of mesothelioma has a characteristic genetic alteration regarding the $\mathrm{p} 53$ expression and the present study suggested that a combinatory use of Ad-delE1B and a p53-augmenting MDM2 inhibitor was a therapeutic strategy for mesothelioma with the wild-type $p 53$ genotype.

\section{REFERENCES}

1. Lee AY, Raz DJ, He B, Jablons DM. Update on the molecular biology of malignant mesothelioma. Cancer. 2007;109:1454-61.

2. Guo G, Chmielecki J, Goparaju C, Heguy A, Dolgalev I, Carbone M, et al. Wholeexome sequencing reveals frequent genetic alterations in BAP1, NF2, CDKN2A, and CUL1 in malignant pleural mesothelioma. Cancer Res. 2015;76:264-9.

3. Bueno R, Stawiski EW, Goldstein LD, Durinck S, de Rienzo A, Modrusan Z, et al. Comprehensive genomic analysis of malignant pleural mesothelioma identifies recurrent mutations, gene fusions and splicing alterations. Nat. Genet. 2016;48:407-16.

4. Tagawa M, Tada Y, Shimada H, Hiroshima K. Gene therapy for malignant mesothelioma: current prospects and challenges. Cancer Gene Ther. 2013:20:150-6.

5. Li Q, Kawamura K, Yamanaka M, Okamoto S, Yang S, Yamauchi S, et al. Upregulated p53 expression activates apoptotic pathways in wild-type p53-bearing mesothelioma and enhances cytotoxicity of cisplatin and pemetrexed. Cancer Gene Ther. 2012;19:218-28

6. Yang S, Kawamura K, Okamoto S, Yamauchi S, Shingyoji M, Senike I, et al. Cytotoxic effects of replication-competent adenoviruses on human esophageal carcinoma are enhanced by forced p53 expression. BMC Cancer. 2015;15:464.

7. Mi J, Li ZY, Ni S, Steinwaerder D, Lieber A. Induced apoptosis supports spread of adenovirus vectors in tumors. Hum. Gene Ther. 2001;12:1343-52.

8. van Beusechem VW, van den Doel PB, Grill J, Pinedo HM, Gerritsen WR. Conditionally replicative adenovirus expressing p53 exhibits enhanced oncolytic potency. Cancer Res. 2002;62:6165-71.

9. Sakai R, Kagawa S, Yamasaki Y, Kojima T, Uno F, Hashimoto Y, et al. Preclinical evaluation of differentially targeting dual virotherapy for human solid cancer. Mol. Canc Ther. 2010;9:1884-93.

10. Larson C, Oronsky B, Scicinski J, Fanger GR, Stirn M, Oronsky A, et al. Going viral: a review of replication-selective oncolytic adenoviruses. Oncotarget. 2015;6:19976-89.

11. Yamanaka M, Tada Y, Kawamura K, Li Q, Okamoto S, Chai K, et al. E1B-55 kDadefective adenoviruses activate p53 in mesothelioma and enhance cytotoxicity of anticancer agents. J Thorac Oncol. 2012;7:1850-7.

12. Hu B, Gilkes DM, Chen J. Efficient p53 activation and apoptosis by simultaneous disruption of binding to MDM2 and MDMX. Cancer Res. 2007;67:8810-7.

13. di Cintio A, di Gennaro E, Budillon A. Restoring p53 function in cancer: novel therapeutic approaches for applying the brakes to tumorigenesis. Recent Pat Anticancer Drug Discov. 2010;5:1-13.
14. Rothmann T, Hengstermann A, Whitaker NJ, Scheffner A, Hausen H. Replication of ONYX-015, a potential anticancer adenovirus, is independent of p53 status in tumor cells. J Virol. 1998;72:9470-8.

15. Yang C-Ta, You L, Uematsu K, Yeh C-C, McCormick F, Jablons DM. p14ARF modulates the cytolytic effect of ONYX-015 in mesothelioma cells with wild-type p53. Cancer Res. 2001;61:5959-63.

16. Bressy C, Hastie E, Grdzelishvili VZ. Combining oncolytic virotherapy with p53 tumor suppressor gene therapy. Mol Ther Oncolytics. 2017;5:20-40.

17. van Beusechem VW, van den Doel PB, Gerritsen WR. Conditionally replicative adenovirus expressing degradation-resistant p53 for enhanced oncolysis of human cancer cells overexpressing murine double minute 2. Mol Cancer Ther. 2005;4:1013-8

18. Yamasaki Y, Tazawa H, Hashimoto $Y$, Kojima T, Kuroda S, Yano S, et al. A novel apoptotic mechanism of genetically engineered adenovirus-mediated tumourspecific p53 overexpression through E1A-dependent p21 and MDM2 suppression. Eur J Cancer. 2012;48:2282-91.

19. Hasei J, Sasaki T, Tazawa H, Osaki S, Yamakawa Y, Kunisada T, et al. Dual programmed cell death pathways induced by p53 transactivation overcome resistance to oncolytic adenovirus in human osteosarcoma cells. Mol Cancer Ther. 2013;12:314-25.

20. Bürck C, Mund A, Berscheminski J, Kieweg L, Müncheberg S, Dobner T, et al. KAP1 is a host restriction factor that promotes human adenovirus E1B-55K SUMO modification. J Virol. 2015;90:930-46.

21. lyengar S, Farnham PJ. KAP1 protein: an enigmatic master regulator of the genome. J Biol Chem. 2011;28:26267-76.

22. Liu H, Naismith JH, Hay RT. Adenovirus DNA replication. Curr Top Microbiol Immunol. 2003;272:131-64.

23. Zhang Q, Cao LY, Cheng SJ, Zhang AM, Jin XS, Li Y. p53-induced microRNA-1246 inhibits the cell growth of human hepatocellular carcinoma cells by targeting NFIB. Oncol Rep. 2015;33:1335-41.

24. Nilsson J, Bjursell G, Kannius-Janson M. Nuclear Jak2 and transcription factor NFIC2: a novel mechanism of prolactin signaling in mammary epithelial cells. Mol Cell Biol. 2006;26:5663-74.

25. Lee JS, Xiao J, Patel P, Schade J, Wang J, Deneen B, et al. A novel tumorpromoting role for nuclear factor IA in glioblastomas is mediated through negative regulation of p53, p21, and PAI1. Neuro-Oncol. 2014;16:191-203.

26. Pjanic M, Pjanic P, Schmid C, Ambrosini G, Gaussin A, Plasari G, et al. Nuclear factor I revealed as family of promoter binding transcription activators. BMC Genomics. 2011;12:181.

27. Wang C, Ivanov A, Chen L, Fredericks WJ, Seto E, Rauscher FJ, et al. MDM2 interaction with nuclear corepressor KAP1 contributes to p53 inactivation. EMBO J. 2005;24:3279-90.

28. Ma G, Shimada H, Hiroshima K, Tada Y, Suzuki N, Tagawa M. Gene medicine for cancer treatment: Commercially available medicine and accumulated clinical data in China. Drug Des Dev Ther. 2008;2:115-22.

29. Hong B, van den Heuvel AP, Prabhu VV, Zhang S, El-Deiry WS. Targeting tumor suppressor 553 for cancer therapy: strategies, challenges and opportunities. Curr Drug Targets. 2014;15:80-89.

30. Tisato V, Voltan R, Gonelli A, Secchiero P, Giorgio ZG. MDM2/X inhibitors under clinical evaluation: perspectives for the management of hematological malignancies and pediatric cancer. J Hematol Oncol. 2017;10:133.

31. Sterman DH, Alley E, Stevenson JP, Friedberg J, Metzger S, Recio A, et al. Pilot and feasibility trial evaluating immuno-gene therapy of malignant mesothelioma using intrapleural delivery of adenovirus-IFNa combined with chemotherapy. Clin Cancer Res. 2016;2:3791-3800.

\section{AUTHOR CONTRIBUTIONS}

TITN prepared the manuscript and the figures and conducted experiments of cytotoxic assay, western blot, and immunofluorescence. MS conducted data processing, statistical analysis, and animal experiments. MH conducted experiments of flow cytometry and animal experiments, and prepared cells treated with agents and adenoviruses. BZ conducted experiments of detecting viral titers and prepared cells treated with siRNA. TM prepared adenoviruses and conducted animal experiments. YT prepared cells and adenoviruses, and designed the experimental protocols. HS conducted data processing and statistical analysis. $\mathrm{KH}$ conducted experiments of immunofluorescence and organized the experiments. MT prepared the manuscript, designed the research directions, organized the experiments, and examined the results.

\section{FUNDING}

This study was supported by Grants-in-Aid from Japan Society for the Promotion of Science (JSPS KAKENHI Grant number: 16K21639, 16K09598, 17K10617, 21K08199) and a Grant-in-aid from the Nichias Corporation. The fund bodies were not involved in the design of the study and collection, analysis, and interpretation of data and in writing the manuscript. 
10

\section{COMPETING INTERESTS}

The authors declare that there is no conflict of interests in this research. We obtained a grant from Nichias Corporation. It is not a pharmaceutical company but a company making industrial products for building, automobiles and pipes (see http://www. nichias.co.jp/). The grant is as a kind of their mécénat activities, corporate social contributions, which is aimed to assist for medical research for intractable cancer treatments. We are thereby irrelevant to any employment, consultancy, patents or products in development or marketed products to the company.

\section{Ethics statement}

This study was approved by the Institutional Ethical Committee and Recombinant DNA committee of Chiba Cancer Center Research Institute and Chiba University (Gene 1-2 and 1-3), and was conducted according to conditions for the use of living modified organisms by Biosafety Regulations in Japan. The animal experiments were approved by the animal experiment and welfare committee of Chiba Cancer Center Research Institute (16-1) and Chiba University (2-302), and were performed according to the guideline on the animal experiments.

\section{ADDITIONAL INFORMATION}

Supplementary information The online version contains supplementary material available at https://doi.org/10.1038/s41419-021-03934-y.
Correspondence and requests for materials should be addressed to M.T.

Reprints and permission information is available at http://www.nature.com/reprints

Publisher's note Springer Nature remains neutral with regard to jurisdictional claims in published maps and institutional affiliations.

(C) Open Access This article is licensed under a Creative Commons Attribution 4.0 International License, which permits use, sharing, adaptation, distribution and reproduction in any medium or format, as long as you give appropriate credit to the original author(s) and the source, provide a link to the Creative Commons license, and indicate if changes were made. The images or other third party material in this article are included in the article's Creative Commons license, unless indicated otherwise in a credit line to the material. If material is not included in the article's Creative Commons license and your intended use is not permitted by statutory regulation or exceeds the permitted use, you will need to obtain permission directly from the copyright holder. To view a copy of this license, visit http://creativecommons. org/licenses/by/4.0/.

(c) The Author(s) 2021 